05;06

\title{
Локальная симметрия канала для транспорта атомов, молекул и его внутренние размеры
}

\author{
(C) Н.А. Крылова ${ }^{1}$, О.В. Клявин ${ }^{2}$, Е.В. Калашников ${ }^{1}$ \\ ${ }^{1}$ Московский государственный областной университет, Москва, Россия \\ ${ }^{2}$ Физико-технический институт им. А.Ф. Иофффе РАН, Санкт-Петербург, \\ Россия \\ E-mail: krylova_nadya_1993@mail.ru
}

Поступило в Редакцию 30 ноября 2017 г.

Обсуждается влияние типа симметрии канала на его внутренние размеры, которые позволяли бы перемещение атомов (ионов, молекул). На основе свойств симметрии функции Лагранжа для канала и свойств самого канала оценены его внутренние размеры. Рассматривается частный пример кристаллографического канала кварца и его дислокаций.

DOI: $10.21883 /$ PJTF.2018.07.45879.17140

Для переноса инородных атомов (ионов, молекул) в различных телах большую роль играют транспортные каналы, поперечные размеры которых сопоставимы или превышают в разы размеры транспортируемых атомов (ионов, молекул) [1-5]. Такими каналами могут быть различные молекулярные структуры в биологии [4], кристаллографические каналы [5] (каналы с открытым типом кристаллографической структуры, например кварц, вода), треки от заряженных частиц, дислокации [1-5]. В частности, проникновение с поверхности и перемещение атомов гелия в кристаллографических телах обусловлены дислокационно-динамической диффузией [6,7]. Транспортные свойства таких каналов определяются тем, как свободно могут в них размещаться и перемещаться атомы (ионы, молекулы). Оценку внутреннего размера канала (ядра дислокации) удается сделать [5,8], но связь структуры канала, его симметрии и его внутреннего диаметра остается неясной.

Цель настоящей работы - оценить внутренние размеры транспортного канала (его радиусы) исходя из его свойств симметрии. 


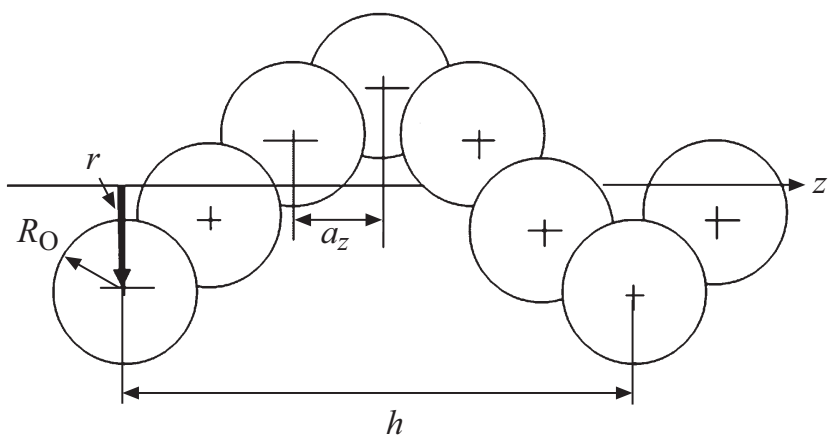

Рис. 1. Кристаллографический канал кварца вдоль направления [0001]. Изображены ионы кислорода, формирующие „внутреннюю стенку“ канала. Каждая пара ионов кислорода принадлежит структурной составляющей кварца тетраэдру $\mathrm{SiO}_{4} . R_{\mathrm{O}}-$ радиус иона кислорода; $a_{z}-$ проекция на ось $z$ расстояний между центрами ионов кислорода в канале; $h-$ период (составлен из шести тетраэдров и равен двум векторам Бюргерса $b) ; r$ - расстояние от оси вращения до центра тяжести иона кислорода, образующего „стенку“ канала.

Независимо от своих функциональных свойств каналы проявляют определенные свойства симметрии. Среди наиболее часто встречаемых типов симметрии каналов можно выделить цилиндрическую и винтовую.

Рассмотрим связь симметрии канала с его внутренними размерами на частном примере кристаллографического канала кварца (рис. 1). Этот канал проявляет свойства винтовой симметрии. Чтобы выявить эти свойства симметрии, рассмотрим функцию Лагранжа $\mathscr{L}$ для канала (в цилиндрической системе координат при условии равноудаленности центров тяжести ионов $r_{j}=r=$ const от оси $z$ ):

$$
\mathscr{L}=\mathscr{L}\left(z_{1}, z_{2}, \ldots, z_{n} ; \varphi_{1}, \varphi_{2}, \ldots, \varphi_{n} ; \dot{z}_{1}, \dot{z}_{2}, \ldots, \dot{z}_{n} ; \dot{\varphi}_{1}, \dot{\varphi}_{2}, \ldots, \dot{\varphi}_{n} ; t\right) .
$$

Здесь $z_{j}-$ смещение, $\varphi_{j}-$ вращение $j$-го атома относительно оси $z$ в канале (рис. 1), верхней точкой обозначены производные по времени $t$ от соответствующих переменных (для канала эти производные равны нулю).

Требование симметрии определенного типа предполагает неизменность функции Лагранжа по отношению к переменным, по которым

Письма в ЖТФ, 2018, том 44, вып. 7 
функция Лагранжа сохраняется (теорема Нётер). Это значит, что при винтовой симметрии вариация функции Лагранжа канала $\delta \mathscr{L}$ при одновременном переносе вдоль оси $z$ (вариации $\delta z$ ) и повороте (вариации) относительно этой оси $(\delta \varphi)$ равна нулю:

$$
\delta \mathscr{L}=\sum_{a}\left(\partial \mathscr{L} / \partial z_{a}\right) \delta z_{a}+\sum_{a}\left(\partial \mathscr{L} / \partial \varphi_{a}\right) \delta \varphi_{a}=0 .
$$

Вариации $\delta z_{a}$ и $\delta \varphi_{a}$ для каждого $a$-го атома одинаковы, конечны и произвольны. Поскольку каждый атом канала (рис. 1) смещается на одно и то же расстояние $\delta z$ и поворачивается на один и тот же угол $\delta \varphi$, имеем

$$
\delta \mathscr{L}=n((\partial \mathscr{L} / \partial z) \delta z+(\partial \mathscr{L} / \partial \varphi) \delta \varphi)=0,
$$

где учтено $z_{n}=z_{1}+n a_{z}$ и $\partial z_{n}=\partial\left(z_{1}+n a_{z}\right)=\partial z$.

В связи с (1) сразу же отметим, что знак вариации лагранжиана роли не играет, но основную роль играет минимальность действия $S=\int_{t_{1}}^{t_{2}} \mathscr{L} d t$ [9]. Это означает, что вариация с положительным или отрицательным знаком неминуемо приведет к увеличению $S$ (кроме того, вариация $\delta \varphi$ проявляет свойства аксиального вектора; знак „минус“ может соответствовать левому винту в правосторонней системе координат). Поэтому выражение (1) запишем в ином виде

$$
\delta \mathscr{L}=n(-(\partial \mathscr{L} / \partial z) \delta z+(\delta \mathscr{L} / \partial \varphi) \delta \varphi)=0 .
$$

Учитывая $\delta z=(h / 2 \pi) \delta \varphi$, получаем, что

$$
-P_{z}(h / 2 \pi)+L_{z}=0
$$

является сохраняющейся величиной. Постоянная интегрирования выбрана равной нулю. Это позволяет избавиться от неопределенности $L_{z}\left(=m r^{2} \dot{\varphi}=m r^{2} \omega\right)$, зависящей от величины $r$, которая и является искомой величиной. Отметим, что если бы мы сохранили вариацию в виде (1), то в (2) получили бы полную положительную форму, а это привело бы к мнимым значениям радиуса канала (3). Поэтому, 
подставляя $L_{z}$ в (2), получим

$$
\begin{aligned}
r & =\sqrt{\left(m v_{z} h / 2 \pi m \omega\right)} \approx \sqrt{(\Delta z / \tau) h / 2 \pi(2 \pi / \tau)} \\
& =\left.(1 / 2 \pi) \sqrt{\Delta z h}\right|_{\Delta z=h}=h / 2 \pi,
\end{aligned}
$$

$r$ - расстояние от оси вращения канала до центра тяжести иона кислорода, образующего „стенку“ канала (рис. 1); $\Delta z$ - смещение вдоль оси $z ; \tau-$ виртуальное время, за которое канал мог бы быть повернут и сдвинут на шаг $h$. Отметим, что здесь можно перейти на „язык“ векторов Бюргерса $b(h=2 b)$ (рис. 1). В модели канала (рис. 1) $h=6 a_{z}$ и

$$
r=6 a_{z} / 2 \pi
$$

Оценим радиус для канала с цилиндрической симметрией. Непосредственно из (1) при условии, что величина $L_{z}$ не определена (2), радиус $r$ оценить не удается (виток должен быть замкнут). Для оценки радиуса канала с цилиндрической симметрией примем, что спираль закручена на одном шаге $a_{z}$, т.е. $h=a_{z}$. В таком случае минимальное значение отношения радиуса канала с винтовой симметрией к радиусу канала $\mathrm{c}$ цилиндрической симметрией равно

$$
\left(6 a_{z} / 2 \pi\right) /\left(a_{z} / 2 \pi\right)=6 .
$$

Значение радиуса (центров тяжести ионов, формирующих внутреннюю „стенку“ канала) для канала с винтовой симметрией (рис. 1) равно

$$
r=6 a_{z} / 2 \pi=6 \cdot 1.83 \AA / 6.28 \approx 1.75 \AA .
$$

Тогда „радиус“ полости канала для винтовой и цилиндрической симметрии равен $r-R_{\mathrm{O}}$ (радиус иона атома кислорода $R_{\mathrm{O}}=1.2 .-1.3 \AA[10]$ ). „Радиус“ полости канала $r-R_{\mathrm{O}}$ (рис. 1) может оказаться положительной или отрицательной величиной

$$
r_{h}=0.55-0.45 \AA, \quad r_{c}=a_{z} / 2 \pi-(1.2-1.3) \AA \approx-(0.9-1.0) \AA .
$$

Из сравнения соотношений (7) радиусов каналов для винтовой и цилиндрической симметрий следует, что канал с цилиндрической симметрией перекрыт ионами кислорода значительно сильнее, чем канал с винтовой симметрией, как видно из рис. 2. Отсюда следует, что

Письма в ЖТФ, 2018, том 44, вып. 7 


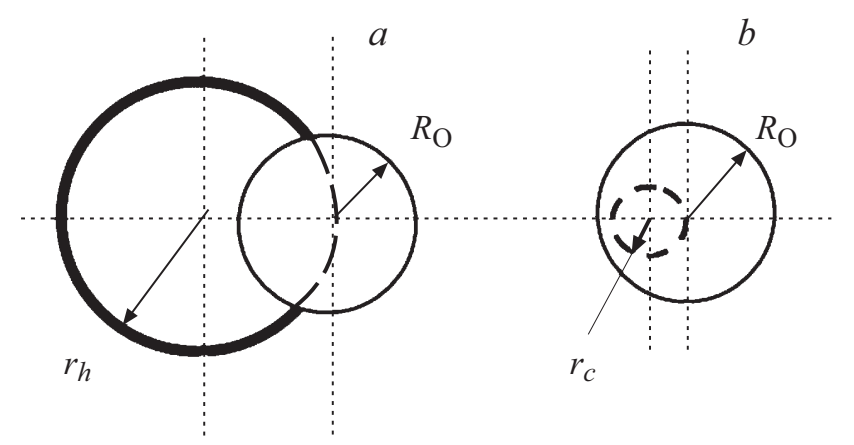

Рис. 2. Перекрытие ионными радиусами кислорода кристаллографического канала: $a-$ с винтовым типом симметрии, $b-$ с цилиндрическим типом симметрии. Штриховой линией обозначена перекрытая часть канала. $R_{\mathrm{O}}-$ paдиус иона кислорода, $r_{h}-$ радиус канала с винтовой симметрией, $r_{c}-$ радиус канала с цилиндрической симметрией.

в канале с винтовой симметрией „свободного места“ для размещения инородного атома больше, чем в канале с цилиндрической симметрией.

Винтовые минимальные дислокации (обеспечивающие минимальную упругую энергию) в кварце располагаются вдоль кристаллографических каналов, растягивая или сжимая их [11]. Для „растянутого“ канала вдоль оси $z$ расстояние $a_{z}$ в (6) и (7) меняется: $a_{z} \Rightarrow(3 / 2) a_{z}$. При этом соотношение (5) не изменится, а „радиусы“ полостей (7) для винтовой и цилиндрической симметрий изменятся следующим образом:

1) для ядра дислокации с винтовой симметрией радиус удаленности центров тяжести ионов кислорода от оси $z$ равен $r=2.65 \AA$, а „радиус“ полости

$$
r_{h}=(3 / 2) \cdot 1.75 \AA-(1.2-1.3) \AA=1.425-1.325 \AA ;
$$

2) для этого же канала в приближении цилиндрической симметрии соответствующие радиусы равны $r=0.291 \AA$ и

$$
\begin{aligned}
r_{c} & =(3 / 2) \cdot a_{z} / 2 \pi-(1.2-1.3) \AA=0.437 \AA-(1.2-1.3) \AA \\
& =-(0.763-0.863) \AA .
\end{aligned}
$$

Таким образом, удалось показать в явном виде влияние типа локальной симметрии транспортного канала на его внутренний радиус

Письма в ЖТФ, 2018, том 44, вып. 7 
$r$ и „радиус“ полости $r_{h}$ и $r_{c}$. Оказалось, что канал с винтовой симметрией обладает внутренним радиусом, значительно превышающим радиус канала с цилиндрической симметрией. Поскольку при выяснении этой ситуации были использованы общие принципы минимальности действия системы, можно предположить, что в большинстве случаев при организации транспортных каналов для переноса атомов (молекул, ионов) предпочтительным типом симметрии будет строение канала, проявляющее свойства винтовой симметрии.

\section{Список литературы}

[1] Sui P., Dai Z. // Sci. China Phys. Mech. Astronomy. 2015. V. 58. N 5. P. $052002(1-6)$.

[2] Ishii A., Li J., Ogata S. // PLoS ONE. 2013. V. 8. N 4. P. e 60586.

[3] Wang L., Drahushuk L.W., Cantley L., Koenig S.P., Liu X., Pellegrino J., Strano M.S., Bunch J.S. // Nature Nanotechnol. 2015. V. 10. N 9. P. 785-790.

[4] Gwan J.-F., Baumgaertner A. // J. Comput. Theor. Nanosci. 2007. V. 4. N 1. P. 50-56.

[5] Калашников Е.В., Толстихин И.Н., Певзнер Б.3. // ФТТ. 2010. Т. 52. В. 7. C. $1283-1290$.

[6] Клявин O.В. Физика пластичности кристаллов при гелиевых температурах. М.: Наука, 1987. 255 c.

[7] Klyavin O.V., Likhodedov N.P., Orlov A.N. // Progr. Surf. Sci. 1990. V. 33. N 4. P. 259-383.

[8] Фридель Ж. Дислокации. М.: Мир, 1967. 643 с.

[9] Ландау Л.Д., Лифиии, Е.М. Механика. М.: Наука, 1988. Т. 1. 215 с.

[10] Pauling $L$. Nature of the chemical bond and structure of molecules and crystals. 2nd ed. Ithaca, N.Y.: Cornell University Press, 1940. 429 p.

[11] Baëta R.D., Ashbe K.H.G. // Am. Mineralogist. 1969. V. 54. N 11-12. P. 15511573.

Письма в ЖТФ, 2018, том 44, вып. 7 